\title{
Link between a local time scale and UTC(OP) by GPS TAIP3 Common-View
}

\author{
G. D. Rovera, ${ }^{1, a}$, B. Chupin ${ }^{1}$, M. Abgrall ${ }^{1}$, and P. Uhrich ${ }^{1}$ \\ ${ }^{1}$ LNE-SYRTE, Observatoire de Paris, PSL Research University, CNRS, Sorbonne Universités, UPMC Univ. Paris 06
}

\begin{abstract}
Résumé. Nous avons mis à point un moyen pour le raccordement d'une échelle de temps locale à UTC(OP), référence légale de temps pour la France. UTC(OP) garantit la traçabilité à la référence internationale UTC via la comparaison clef CCTF-K001.UTC. Les données, en format CGGTTS, d'un récepteur GPS de type géodésique connecté à l'échelle de temps locale sont comparées avec les données au même format des récepteurs GPS du LNE-SYRTE. Le logiciel pour le calcul de la différence entre les deux échelles de temps est distribué sous licence open-source de type CECIL. Les données journalières du LNE-SYRTE et le logiciel peuvent être récupérés à partir du site web du LNE-SYRTE. L'incertitude du raccordement dépend principalement de l'incertitude des délais de la station GPS locale. Ce délais peuvent être connu au niveau de quelque ns avec un étalonnage de haut niveau [1] ou de quelques centaines de ns pour une station non étalonnée. L'article décrit en détail les performances du logiciel et présente les résultats obtenus en comparant UTC(OP) à d'autres échelles de temps réalisées dans d'autres laboratoires.
\end{abstract}

\section{Introduction}

The LNE-SYRTE in Observatoire de Paris (OP), Paris, France, is the institute designated by the French National Metrology Institute (NMI), Laboratoire National de Métrologie et d'Essais (LNE), in the field of Time and Frequency metrology. In this frame, the laboratory has a long history in developing remote clock comparisons by using the well-known Common-View (CV) technique on the signals broadcast by the Global Positioning System (GPS). In particular, OP has been using for more than ten years data files in the Common GNSS Time Transfer Standard (CGTTS) format based on the ionosphere free linear combination of GPS P-Code pseudo-ranges collected on both L1 and L 2 carriers by geodetic receivers, namely the TAIP3 technique [2]. To improve the time transfer short term stability, OP had also developed a technique based on the filtering of $\mathrm{CV}$ around a mean value computed for each CGTTS epoch, which are sampled on 16 min periods, in order to average the outlier out [3]. Through the years, other teams have also proposed some averaging techniques like for instance in [4].

In this paper, we present a simple averaging technique, aiming at improving the short term stability together with the robustness of GPS CV from TAIP3 data files [5]. The software implementing this technique, has been intensively tested in routine comparison of UTC(OP) with the UTC(k) of a few NMI. It is now released with an open-source license and is available on LNE-SYRTE website [6]. We analyze the results obtained over two different kinds of baselines: one short baseline between OP and the Physikalisch Technisches Bundesanstalt (PTB),

\footnotetext{
a e-mail: daniele.rovera@obspm.fr
}

Braunschweig, Germany, and one long baseline between OP and the United States Naval Observatory (USNO), Washington DC, USA.

\section{The New Averaging Technique}

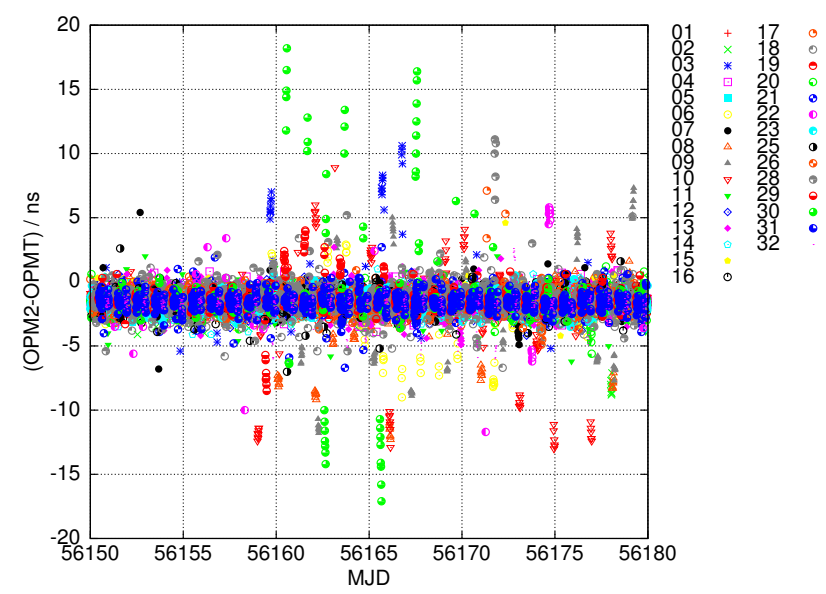

Figure 1. Raw TAIP3 CV data between OPMT and OPM 2 implemented in OP in a common-clock and common-antenna setup. The corresponding GPS SV number symbols are given on the right of the figure.

LNE-SYRTE is operating continuously two geodetic GPS receivers in a common-clock and common-antenna set-up. The receivers are called OPMT and OPM2, and this ensemble is the basis of the International GNSS Service (IGS) station located in OP called OPMT. When looking at the TAIP3 CV between both receivers, we noticed 
that there were epochs where some given satellite data were outside the mainstream of other data. Figure 1 shows the TAIP3 CV between OPMT and OPM2 over about $30 \mathrm{~d}$ of data, for all GPS satellites tracked, with a different symbol for each satellite. This is a typical plot for this set-up, which is in operation for more than ten years now. In that case, the epoch filtering might sometimes lead to biased mean values, increasing that way the short term noise of the time transfer.

From there we decided to try to improve the computation of the CV mean values by averaging out the kind of satellite outliers we see in Figure 1. We start from the CGTTS GPS TAIP3 files collected by two different receivers, and we compute $\mathrm{CV}$ daily files. We then compute a linear fit over one day of CV data, and we average out all 3 sigma outliers in an iterative process. We tested different cycle iterations, but we finally obtained the best results with an iterative process without any limitation. This allows to suppress either "bad" CV due to some problems in the reception of a given satellite signal, or CV exhibiting abnormal high noise, or any unusual outliers from a biased satellite. Back to the original CGTTS TAIP3 CV data files, we compute a mean value between the remaining CV for each common CGTTS epoch, leading to the time transfer results for the reference time scales. Figure 2 shows the processing results on the TAIP3 CV between OPMT and OPM2 over the same period of time as in Figure 1. The direct comparison to Figure 1 is providing one obvious aspect of the improvement, the dispersion of the data after daily filtering being limited to a little more than $3 \mathrm{~ns}$ peak to peak. Note that the quantified distribution of the filtered CV is only caused by the fact that the tracking measurement resolution is limited to $0.1 \mathrm{~ns}$ in the CGTTS data format.

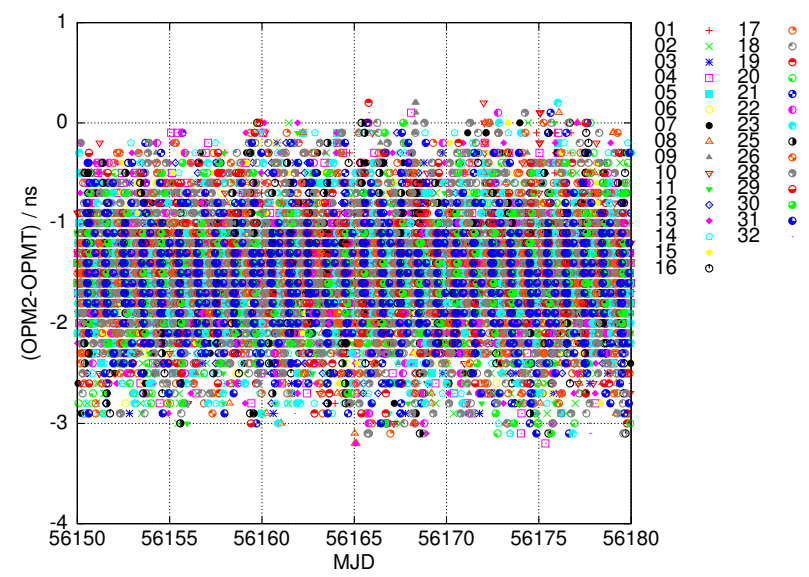

Figure 2. TAIP3 CV between OPMT and OPM2, after application of the new filtering approach. The corresponding GPS SV number symbols are on the right of the figure.

The analysis of this new technique is based on two different criteria. First, we compare the average number of remaining satellites between the former OP CV computation and the new daily approach on time transfer data. In the case the new technique would average out less satellite $\mathrm{CV}$ compared to the former one, it would show an increase of robustness in the time transfer link. And second, we compute the Allan Time Deviation (TDEV) of the $\mathrm{CV}$, obtained either from the former OP epoch filtering or with the new daily approach for the same period of time between two stations. We expect there should be some consistency between both criteria if the short term noise is white, as an average increase in the remaining $\mathrm{CV}$ by a factor $\mathrm{n}$ should lead to a decrease in the TDEV by a factor $\sqrt{n}$. We have applied this technique on the TAIP3 CV between OPMT and OPM2. The remaining number of satellites left after filtering is about $82 \%$ larger for the daily new approach compared to the former epoch filtering, increasing that way the robustness of the resulting CV. Figure 3 shows the TDEV of the OPM2 - OPMT $\mathrm{CV}$ for three different data sets. In blue, the TDEV of raw data is plotted. A very small number of data have already been filtered out here, either because the figures were obviously not related to any measurement with GPS satellites, or because of some technical problems in OP for that given epoch. What appears as a large periodic effect in this TDEV is related to some regular and irregular outliers, which are precisely the outliers we wish to suppress. In red, the TDEV of the former epoch filtering developed by OP [3]. The outliers having been averaged out, the TDEV exhibits a white phase noise modulation (slope -1/2) up to an averaging period of about $2 \mathrm{~d}$, where the flicker floor of the common receivers' noise is limiting the stability to between 20 and 30 ps. And in green, the TDEV of the new daily approach shows from the sampling period on an improvement by a factor 1.37 , which is completely consistent with the increased number of $\mathrm{CV}$ after filtering, as we get $\sqrt{1.82}=1.35$. It confirms the white phase noise modulation which dominates until the flicker floor of both receivers noise.

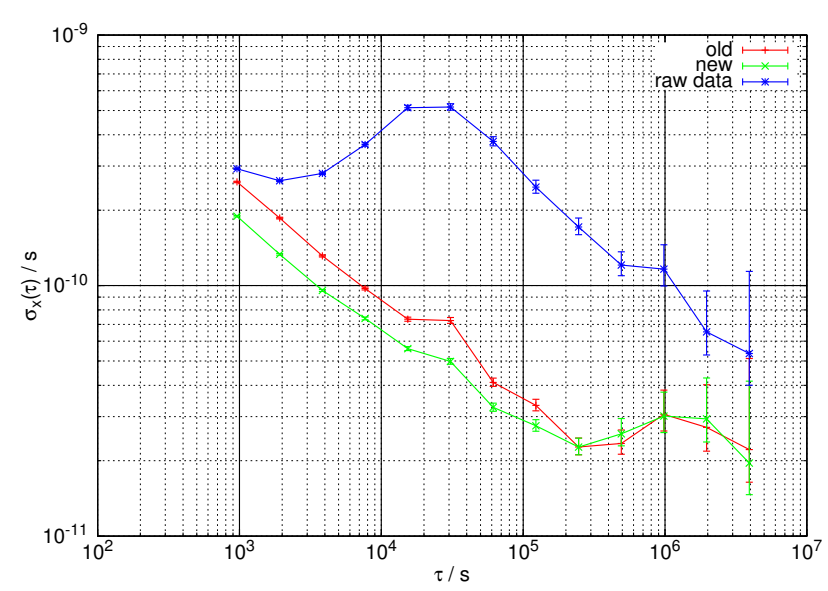

Figure 3. Allan Time Deviation (TDEV) of the TAIP3 CV between OPMT and OPM2 in a common-clock and common antenna set-up. In blue, the original raw data. In red the former epoch filtering OP process. And in green the new daily approach.

To analyze what happens between remote $\mathrm{UTC}(\mathrm{k})$ time scales, we have applied this new daily filtering approach on two different links. In Section 3, we show the results obtained over the short baseline of around $1000 \mathrm{~km}$ 
between OP and PTB. In 3, we show the results over a long baseline of more than $6000 \mathrm{~km}$ between OP and USNO.

\section{The OP - PTB Link}

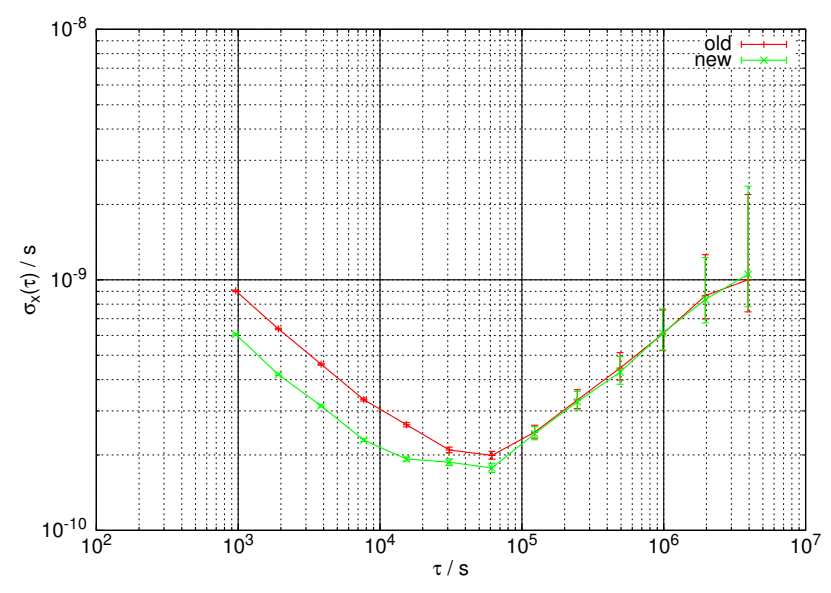

Figure 4. Time Allan Deviation (TDEV) of the TAIP3 CV between OP and PTB: the former OP epoch filtering results are in red, the new daily approach results are in green.

This GPS link is of particular importance for LNESYRTE, because it may be used by the Bureau International des Poids et Mesures (BIPM) for its computation of Temps Atomique International (TAI), leading to the Key Comparison UTC - UTC(OP) [5]. We have used TAIP3 data collected in remote stations for more than $200 \mathrm{~d}$. The average number of remaining satellite $\mathrm{CV}$ after filtering of the outliers through the new daily approach appears to be $81 \%$ larger than the CV number obtained from the former epoch filtering technique. Figure 4 shows the TDEV obtained from filtered CV between OP and PTB, either when using the former averaging software (in red) or with the new daily approach (in green). As expected over such a short baseline, because of numerous satellites available to compute the $\mathrm{CV}$, the short term noise is very close to a white phase noise modulation, as can be seen in the ${ }^{-} 1 / 2$ slope, until the time scale noise becomes higher. Therefore, the short term stability should be improved by a factor close to $\sqrt{1.81}=1.35$.

We gain 1.49 on the TDEV here from the sampling period on, which is a little larger than expected, but does not question the consistency of both results. It only indicates that the filter quality was also improved, and this is discussed in more detail in the next Section. A noise below $0.2 \mathrm{~ns}$ can be reached just above an averaging period of $10000 \mathrm{~s}$, where the asymptotic behavior of the plot is limited by the time scale noise.

These results are showing a clear improvement over short baselines when using the automated new daily filtering approach compared to the former OP computation based on epoch filtering. Next Section discusses the example of a long baseline.

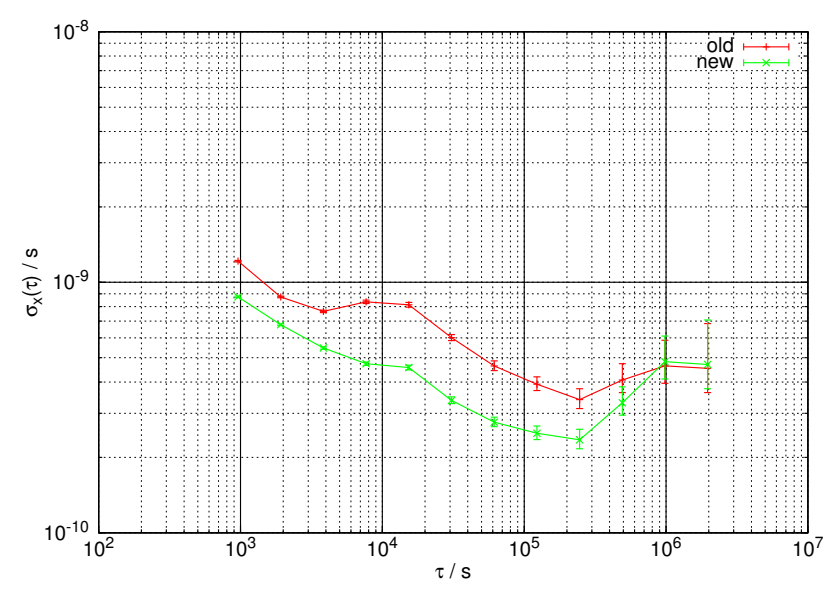

Figure 5. Time Allan Deviation (TDEV) of the TAIP3 CV between OP and USNO: the former OP epoch filtering results are in red, the new daily approach results are in green.

\section{The OP - USNO Link}

Here too, we have used TAIP3 data collected in remote stations for more than $200 \mathrm{~d}$. The average number of remaining satellite $\mathrm{CV}$ after filtering of the outliers through the new approach appears to be $23 \%$ larger compared to the CV number obtained from the former technique. But it has to be remembered that over such a large baseline, the number of available CV stays low compared to a continental link.

Figure 5 shows the TDEV obtained from filtered CV between OP and USNO, either when using the former epoch averaging OP software (in red) or with the new daily filtering approach (in green). In the case the phase noise modulation would be white, the improvement on the TDEV first points from sampling period on should be about $11 \%$. We gain a factor 1.38 on the first TDEV point at the sampling period, and 1.29 on the second TDEV point, which is again larger than expected. But before reaching an averaging period of $10000 \mathrm{~s}$, the noise of the data filtered by the former OP epoch processing seems affected by an unexpected modulation, leading to a difference close to a factor 2 between that TDEV and the new daily filtering approach up to an averaging period above 1 d. The noise of the time scales becomes higher than the transfer noise above an averaging period of about $5 \mathrm{~d}$ and the two curves are almost superposed.

After analysis, it appears that the large unexpected TDEV improvement between $10000 \mathrm{~s}$ and $1 \mathrm{~d}$ is related to a singularity in the TAIP3 CV data. For some unknown reason, during a few consecutive CGTTS epochs, the TAIP3 CV raw data were biased on some satellite measurements which were not in line with the time transfer results obtained before and after that singularity. Figure 6 shows the event on the filtered results on CGTTS epoch mean values. In Figure 6, the mean values issued from an epoch averaging of the raw data are in blue. During the singularity event the mean values are located between the mainstream data before and after the event and some large outliers which CV are far above $40 \mathrm{~ns}$ away. But be- 


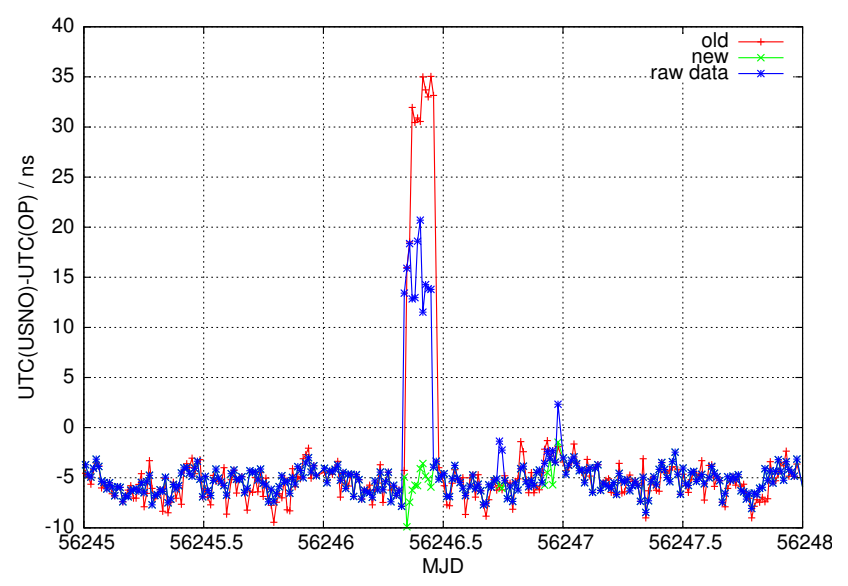

Figure 6. Raw TAIP 3 CV data between OPMT and OPM 2 implemented in OP in a common-clock and common-antenna setup. The corresponding GPS SV number symbols are given on the right of the figure.

cause there were numerous satellites involved, the former OP epoch averaging technique provides as mean value an inconsistent result in red. The former epoch filtering gives too much credit to the outliers, and the $\mathrm{CV}$ averages are not in line with the other CV mainstream results. In the contrary, the new daily approach in green consistently averages out the outliers, and provides the expected results in line with the CV mainstream results. We assume that this happens even for small outliers, and this explains why the improvements on the TDEV are larger than expected when looking at the increase of CV numbers. Clearly, the new approach provides more stable and more robust results than the former OP processing.

\section{Conclusion}

In this paper, we propose a new simple averaging technique for TAIP3 GPS CV which improves both the stability and the robustness of time transfer between remote stations. Instead of averaging the outliers out by using a given filter around a mean value computed for each CGTTS sampling epoch, we average the outliers out from a linear fit on daily CV files. Between two GPS receivers in commonclock and common antenna set-up, the number of available CV left after filtering is $82 \%$ larger than with the former epoch filtering developed by OP. The white phase noise improvement on a TDEV plot is consistently in line with that result, from the sampling period on until the flicker floor limit of the receivers noise. Over a short baseline of less than $1000 \mathrm{~km}$ between remote stations, we obtain a similar increase in the $\mathrm{CV}$ number after filtering, but the TDEV improvement is slightly larger than expected. Over a long baseline of more than $6000 \mathrm{~km}$, the TDEV improvement is also much larger than expected, but this can be explained by the way the former OP epoch processing was giving too much credit to some outliers compared to the data mainstream. The new daily approach gives more consistent results, improving that way both the short term stability and the robustness of time transfer in all cases. This new processing approach has been implemented in OP in a completely automated way. As being a very simple filtering technique, we expect it might be useful in the future for any GNSS time transfer based on code data. The software has been intensively tested in routine comparison. Although originally developed for a Linux system the software has also been tested in several flavors of Windows. The graphical interface allows the interactive operation, but also produces a XML file containing the manually chosen configuration. The configuration file can be saved and used to perform automated comparisons.

The system presented in this paper allows the traceability of the local time scale to UTC(OP) with an uncertainty dominated by the uncertainty of the internal delays of the GPS receiver system. It has been demonstrated that with a careful calibration on a particular link an uncertainty of a few ns can be reached [1]. In the general case of uncalibrated GPS receiver an accuracy in the range of hundreds of nanosecond can be expected. When the user only needs frequency traceability the calibration of the receiver is unnecessary and the only requirement is the stability of the delays, in this case the accuracy will be limited only by the noise of the link and of the local oscillators.

\section{Acknowledgement}

The authors are grateful to P. Defraigne from Observatoire Royal de Belgique (ORB) for having made her TAIP3 software freely available to the Time Metrology community.

\section{References}

[1] G.D. Rovera, et al. Link calibration against receiver calibration time transfer uncertainty when using the Global Positioning System Metrologia 51, 476 (2014)

[2] P. Defraigne, G. Petit, Time transfer to TAI using geodetic receivers Metrologia 40, 184 (2003)

[3] J. Achkar, P. Uhrich, P. Merck, D. Valat, Recent time and frequency transfer activities at the Observatoire de Paris, in Frequency Control Symposium, 2005. Proceedings of the 2005 IEEE International (2005), pp. 247-253

[4] R. Costa, D. Orgiazzi, V. Pettiti, I. Sesia, P. Tavella, Performance comparison and stability characterization of timing and geodetic GPS receivers at IEN, in Frequency and Time Forum, 2004. EFTF 2004. 18th European (2004), pp. 279-286

[5] G. Rovera, B. Chupin, M. Abgrall, P. Uhrich, A simple computation technique for improving the short term stability and the robustness of GPS TAIP3 commonviews, in European Frequency and Time Forum International Frequency Control Symposium (EFTF/IFCS), 2013 Joint (2013), pp. 827-830

[6] https://syrte.obspm. fr/tfc/temps/ outgoing_data/gnss 